\title{
Labyrinthe
}

\section{Communauté/groupe ethnique}

Des impératifs de sécurité nationale aux pratiques sociales délimitant les frontières du groupe

Jeanne Hersant

\section{(2) OpenEdition}

1 Journals

Édition électronique

URL : http://journals.openedition.org/labyrinthe/914

DOI : $10.4000 /$ labyrinthe.914

ISSN : 1950-6031

Éditeur

Hermann

Édition imprimée

Date de publication : 22 juin 2005

Pagination : 95-102

Référence électronique

Jeanne Hersant, « Communauté/groupe ethnique », Labyrinthe [En ligne], 21 | 2005 (2), mis en ligne le 15 juillet 2008, consulté le 01 mai 2019. URL : http://journals.openedition.org/labyrinthe/914 ; DOI :

10.4000/labyrinthe.914 


\section{COMMUNAUTÉ/GROUPE ETHNIQUE \\ Des impératifs de sécurité nationale aux pratiques sociales délimitant les frontières du groupe}

Jeanne HERSANT

Pourquoi et comment passer de la communauté au groupe ethnique? La transition entre les deux notions est souvent implicite dans les ouvrages en sciences sociales, et formulée comme allant de soi, aussi vaut-il mieux la clarifier. J'ai déjà mentionné plus haut le rapprochement opéré par Thomas Faist en ce sens ${ }^{1}$, je me référerai ici à la définition de la communauté donnée par Riva Kastoryano:

La sociologie classique présente la communauté (Gemeinschaft) comme une forme d'organisation traditionnelle et statique caractérisée par la proximité des relations sociales, fondées sur la «volonté naturelle» ou «spontanée». De ce fait, elle s'oppose à la société (Gesellschaft) où c'est une volonté «réfléchie», donc la rationalité, qui détermine les relations entre individus. Dans la même logique les individus membres d'une communauté définissent leur attachement à celle-ci par des liens «primordiaux », i.e. des traits d'identité qu'ils partagent dès leur naissance (liens de sang, religion, unité linguistique, etc.) et qui constituent leur «identité de base².»

Je ne reprendrai pas à mon compte cette définition qui ne me paraît pas clarifier la distinction (ou l'absence de distinction) entre communauté et groupe ethnique, d'autant que l'auteur, dans un autre passage, utilise indistinctement les termes «communauté ethnique» (à propos des minorités), «communauté nationale» (à propos de la société majo-

\footnotetext{
1. «The Border-Crossing Expansion of Social Space: Concepts, Questions and Topics», dans Thomas Faist et Eyüp Özveren (dir.) op. cit.

2. «Construction de communautés et négociations des identités. Les migrants musulmans en France et en Allemagne», dans Denis-Constant Martin (dir.), Cartes d'identité. Comment dit-on «nous » en politique?, Paris, FNSP, p. 230. Cette définition est en partie reprise de celle de F. Tönnies. $\rightarrow$ «Sciences sociales: définitions et traditions» $\nleftarrow$
} 
ritaire et de l'État d'accueil), et «communauté politique» (pour la classe politique et les décideurs). Je m'en tiendrai à la distinction établie précédemment $\rightarrow$ «Réflexion sur les catégories du droit international...» : la communauté équivaut ici au groupe ethnique dont elle est la projection intellectuelle et affective. J'orienterai plutôt mon analyse suivant une autre proposition de Kastoryano:

La frontière des communautés ainsi que leur contenu identitaire changent par l'effet des interactions avec les autres groupes et la société globale (Barth, 1969). C'est notamment au cours d'un processus de politisation que le groupe ou la communauté sont conduits à définir des frontières (Kastoryano, $1994: 230$ ).

La politisation - au sens que lui donne Jacques Lagroye ${ }^{3}$ - est en effet un point central : elle est ce qui incite les membres d'un groupe à marquer leur différence vis-à-vis des autres, particulièrement si cette différence, comme dans le cas de la Thrace, leur procure une prédominance politique et sociale au regard des autres populations minoritaires, fût-elle relative par rapport au groupe grec majoritaire. La politisation est également ce qui permet de faire le lien entre la communauté au sens institutionnel et la communauté «imaginée». Je m'explique: si des comportements collectifs distinctifs - notamment du point de vue démographique - peuvent survivre à la disparition d'institutions englobantes socialement ancrées ${ }^{4}$, ces comportements distinctifs n'aboutiront pas pour autant à la constitution d'un groupe car il y manquera la dimension de stratégie identitaire, le travail intellectuel et/ou idéologique visant à donner du sens à ces comportements, propre aux processus d'ethnicisation.

3. «[Il s'agit de] comprendre comment [...] des rapports sociaux, des styles de relations, des formes d'échange et de communication, des pratiques professionnelles, des engagements associatifs et ainsi de suite, deviennent des éléments ou des règles de l'espace politique et produisent de surcroît les catégories de pensée qui permettent d'en parler. Il s'agit là des formes et des voies d'une conversion, celle de toutes sortes de pratiques en activités politiques. C'est cette conversion qu'on appelle ici politisation. En retour, la politisation peut désigner l'infinie diversité des effets de cette conversion sur les acteurs et les activités apparemment les plus éloignés du jeu politique, les plus étrangers à ses règles et à ses enjeux [...]», Jacques Lagroye, «Avant-propos», dans La Politisation, Paris, Belin, 2003.

4. Morgane Labbé, op. cit. 


\section{Communauté/groupe ethnique}

Ce constat rejoint l'idée d'Olivier Roy selon laquelle l'ethnicité peut être une construction «par le haut» et qu'elle ne saurait être, dès lors, dissociée des dynamiques politiques à l'œuvre dans le contexte étudié :

Le passage des groupes d'identités primaires à ce que nous appellerons des macro-ethnies, où le critère de la langue devient primordial, éventuellement couplé avec celui de la religion [...], entraînant une revendication de territorialisation, est en général induit par un processus politique, qu'il vienne d'une volonté étatique ou tout simplement de l'auto-reclassement des groupes de solidarité confrontés à des pressions extérieures et à la nécessité de s'affirmer dans des cadres identitaires plus larges que celui de leur groupe originel [...]. Or, c'est au niveau de telles macro-ethnies que s'articulent les stratégies d'État, ne serait-ce que parce qu'un État, pour définir sa stratégie régionale, ne peut pas intervenir sur des ensembles flous et démographiquement trop peu importants 5 .

On peut certes émettre des réserves sur l'habitude des chercheurs de poser des «critères primordiaux» qui permettent d'identifier un groupe, ou sur l'emploi d'un lexique encore et toujours primordialiste («groupe originel»). Quoi qu'il en soit, retenons l'idée selon laquelle la stratégie étatique et le processus politique (impliquant donc des rapports de et une course au pouvoir) ont été suffisamment performants pour entraîner des recompositions identitaires et des aménagements dans les pratiques sociales. Lorsqu'une telle construction est efficace, en effet, elle finit par acquérir du sens auprès des acteurs concernés qui se réapproprient les démarcations ethniques.

La minorité «turque» en Thrace occidentale s'est vue de la sorte imposer une identité «par le haut»: par les autorités grecques d'une part, qui l'ont délibérément placée en dehors du processus de construction nationale, et par l'État turc, dans le cadre des prérogatives qui lui furent peu à peu attribuées en pratique. Cette présence turque s'est manifestée par l'ingérence institutionnelle, on l'a vu $(\leftrightarrow \ll$ Communauté/minorité...» $\nleftarrow)$; je décrirai ici les pratiques sociales d'identification à la «macro-ethnie» turque au sein de la minorité

5. «Ethnies et politique en Asie centrale», REMMM, «Des ethnies aux nations en Asie centrale», $1991,59-60(1-2)$, p. 22-23. 
musulmane de Thrace occidentale. Mais voyons tout d'abord la genèse de cette politisation des appartenances et pratiques sociales.

\section{Ethnicisation «par le haut»}

Depuis les années 1960, avec la prise en charge progressive par le consulat de Turquie des salaires du fonctionnariat autochtone (principalement le personnel religieux et les instituteurs $)^{6}$, au sein de la minorité s'est progressivement imposée l'idée que l'ascension sociale passe par la Turquie: soit par l'intermédiaire de sa représentation sur le territoire grec, soit par un parcours scolaire/universitaire ou des investissements immobiliers en territoire turc. De façon concomitante, la socialisation des élites locales au contact des diplomates turcs, mais aussi leur formation universitaire en Turquie, a favorisé le glissement de la minorité vers une catégorisation «ethnique», en l'occurrence turque.

La cession de telles prérogatives aux autorités turques fut rendue possible par le fait que les autorités grecques considéraient cette minorité comme «turque » : cette simplification en facilitait la gestion (d'autant que la minorité «musulmane » était jugée de toute façon inassimilable à la nation grecque), en coopération avec la Turquie alliée contre le danger communiste, dans le cadre de l'Otan. Rappelons que la composante pomaque de cette minorité parle un dialecte proche du bulgare, et vit le long de la frontière gréco-bulgare. D'ailleurs, la Grèce, et particulièrement cette frontière, fut longtemps considérée comme «le rideau de fer des Balkans ${ }^{7}$ »; il s'imposait donc d'empêcher, par tout moyen, que cette population bulgarophone entre en contact avec l'ennemi communiste, et il valait mieux en assurer le contrôle, conjointement avec la Turquie. Depuis les années 1980 particulièrement, alors que le contentieux chypriote redéfinissait les impératifs régionaux de sécurité, les autorités grecques ont adopté une position inverse, en refusant de reconnaître une minorité autre que musulmane, et en promou-

\footnotetext{
6. Depuis les années 1960, les instituteurs des écoles de la minorité sont en grande partie rémunérés par le consulat turc de Komotini, et depuis la fin des années 1980 ce sont les muftis et une partie du personnel religieux, à quoi s'ajoutent les subventions allouées aux associations et à la presse locale turcophone.

7. D'ailleurs, toute la zone de peuplement pomaque fut, particulièrement au nord de Xanthi (İskeçe), jusqu'en 1998, une zone militaire fermée.
} 
vant l'affirmation identitaire de la composante pomaque de cette minorité, dont les membres ont tendance à se dire turcs plutôt que pomaques, identité fortement stigmatisée car associée au sous-développement économique de l'ancienne zone interdite.

S'intéresser à cette marge permet de mieux appréhender les réajustements en cours, en considérant que la «frontière» nécessaire à la délimitation du groupe ethnique peut avoir la signification de «marge». En effet, c'est toujours à la marge, dans l'entre-deux, que se jouent la définition et la normalisation des groupes comme des principes qui les régissent: la marge, c'est-à-dire les villages en général, en raison de la hiérarchisation sociale opérée autour de la dichotomie rural/urbain $(\leftrightarrow$ «Communauté/minorité...» ), mais plus précisément les villages de l'ancienne zone militaire fermée, la région de peuplement pomaque au nord de Xanthi, près de la frontière bulgare. La composante pomaque de la minorité «musulmane» est le point faible du groupe ethnique turc, parce qu'elle est jugée insuffisamment turcophone, et surtout insuffisamment intégrée aux réseaux sociaux turcs, essentiellement urbains comme on l'a vu. Ainsi, ce sont toujours les électeurs de ces villages, soupçonnés de collaboration avec les Grecs, qui sont montrés du doigt après chaque élection, lorsqu'il apparaît que l'électorat musulman n'a pas pu élire le nombre de députés auquel son importance numérique lui donnait droit ${ }^{8}$.

Il a été démontré que cette région a été volontairement isolée et laissée à l'écart du développement économique régional ${ }^{9}$. La permanence du clivage ville/village continue de rendre performant le modèle intégrateur «turc », associé à la modernité et à l'ascension sociale, comme l'illustre notamment le fait que la plupart de mes jeunes interlocutrices quittant leur village pour la ville, dans la région de Xanthi, abandonnent le foulard, attribut typiquement villageois. Une de mes interlocutrices du quartier de Zeytinburnu à Istanbul, qui a grandi à Xanthi (İskeçe) et dont la famille est originaire du village d'Oreon (Yassiören) dans la zone de peuplement pomaque, était désolée que j'aie mené des entretiens dans un des villages proches du sien, parmi ces gens «qui

\footnotetext{
8. Jeanne Hersant, Nepheli Yatropoulou et Yannis Bonos, «Le facteur turc dans la vie politique locale en Thrace occidentale», Les Dossiers de l'IFEA, à paraître en 2005.

9. Lois Labrianidis, «The Impact of the Greek Military Surveillance Zone on the Greek Side of the Bulgarian-Greek Borderlands », Boundary and Security Bulletin, 1999, 7 (2), p. 82-93; «Internal Frontiers as a Hindrance to Development», European Planning Studies, 2001, 9 (1), p. 85-103.
} 
ne savent rien». Elle refuse quant à elle qu'on la nomme «pomaque» pour la raison suivante: «Nous sommes de vieux Xanthiotes (İskeçeli), ma mère est venue en ville quand elle était très jeune, et elle fut l'une des premières à cesser de porter le voile ${ }^{10}$.»

\section{Le maintien des frontières du groupe malgré l'ouverture politique et sociale}

Auparavant, même si «Grecs» et «Turcs» vivaient côte à côte, le système scolaire (particulièrement l'université) ${ }^{11}$ et la fonction publique grecs étaient fermés ou inaccessibles aux membres de la minorité, de même que la représentation politique à l'échelon local. Tout cela a volé en éclats au cours de la décennie 1990, et surtout dans la seconde moitié de cette décennie, sous l'impulsion européenne ${ }^{12}$. Se pose alors la question du maintien des frontières du groupe, dont les relations avec la société majoritaire sont en voie de normalisation.

Au cours des discussions auxquelles j' ai pris part avec des «Turcs», en Thrace occidentale, le recours à l'opposition «eux/nous » est quasi systématique. «Eux», ce sont les «Grecs», les riches, les détenteurs du pouvoir, voire les oppresseurs. Cette opposition binaire totalisante se retrouve aussi dans la presse locale ${ }^{13}$, à travers les termes minorité/majorité, opposition reprise opportunément par les hommes politiques «grecs» locaux, ce qui leur évite d'avoir à prononcer l'adjectif « turc $^{14} »$. Ainsi la dénonciation des inégalités, discriminations et oppressions - réelles jusqu'aux années 1990, beaucoup moins évidentes aujourd'hui malgré une suspicion diffuse et récurrente à

\footnotetext{
10. Entretien à Zeytinburnu (Istanbul), février 2004.

11. Hormis les difficultés liées à la sélectivité du système grec, un de mes interlocuteurs de Xanthi, ayant effectué son parcours universitaire à Istanbul, puis à Londres, m'expliqua que jusque dans les années 1990 « [pour eux], c'était naturel d'aller en Turquie»; il ne lui serait pas venu à l'idée et cela aurait semblé aussi incongru à ses parents de l'inscrire dans une université grecque que de l'envoyer «à l'étranger». Entretien à Xanthi (İskeçe), septembre 2003.

12. Jeanne Hersant, «La minorité musulmane en Thrace et l'intégration européenne de la Grèce », art. cit.

13. Je travaille notamment à partir d'une étude de presse du journal Gündem (ordre du jour) de Komotini, entre septembre 2002 et septembre 2004.

14. Jeanne Hersant, Nepheli Yatropoulou et Yannis Bonos, art. cit.
} 
l'égard des «Turcs»-, s'efface parfois devant le repli sur la défense des institutions communautaires.

Hormis le clivage discursif, c'est en effet le spectre de l'assimilation à la société grecque qui mobilise le plus. Dans cette optique, l'ouverture croissante des institutions, notamment des universités grecques, aux membres de la minorité (il existe un quota pour les étudiants musulmans de la Thrace depuis 1996), serait un stratagème pour éloigner les jeunes de leur famille et de l'environnement minoritaire, afin qu'ils perdent leurs repères et leurs valeurs; et surtout afin qu'ils échappent au contrôle social communautaire. La conséquence en serait la généralisation des mariages mixtes, qui constituent selon la plupart de mes interlocuteurs un danger pour la survie de la présence turque. L'interdit matrimonial n'est en soi pas nouveau; Jean-François Gossiaux rappelle que, à l'instar de la structure de parenté qui repose sur l'interdit de l'inceste, la structure ethnique repose elle-aussi sur un interdit matrimonial, qui concerne l'exogamie ${ }^{15}$. La nouveauté est à chercher du côté de l'intellectualisation de cet interdit, qui trouve sa place et justification dans le discours de résistance à la stratégie assimilatrice grecque.

En dépit de ces marqueurs identitaires performants puisque décelables jusque dans les conduites quotidiennes, l'élément suivant nous permet de dissocier le groupe ethnique de la communauté: bien qu'il y ait une politisation évidente de l'identité turque, il n'y a pas pour autant de comportement politique collectif communautaire comme le montre l'échec récurrent des appels au vote communautaire de la part des leaders « turcs» lors des différents scrutins en Thrace occidentale ${ }^{16}$. Les résultats électoraux montrent une intégration croissante de l'électorat musulman au système politique grec, alors même que, partageant désormais la logique communautaire de leurs homologues «turcs », les candidats «grecs» eux-mêmes exhortent les «musulmans» (certains vont même jusqu'à promouvoir l'identité turque de la minorité) à voter pour «leurs propres » candidats, c'est-à-dire ceux de la minorité ${ }^{17}$.

Lorsque l'on passe de la structure communautaire confessionnelle au groupe ethnique, dans le cas de la Thrace occidentale, le principe

15. Jean-François Gossiaux, séminaire «Anthropologie des espaces politiques », EHESS, 15.11.04. 16. Lors de la campagne des élections législatives de mars 2004, un collaborateur du journal Gündem (également propriétaire du lycée de la minorité de Xanthi) réclama dans un éditorial le retour aux collèges électoraux distincts pour les «Grecs » et les «Turcs».

17. Jeanne Hersant, Nepheli Yatropoulou et Yannis Bonos, art. cit. 


\section{Labyrinthe, $n^{\circ} 21$}

d'organisation sociale de la minorité reste le même, marqué à la fois par des institutions même désuètes, et par une forte politisation de l'identité. Ses frontières se déterminent en fonction d'un double principe d'organisation sociale. Il faut donc envisager parallèlement le processus interne (relative stabilité de l'organisation sociale hiérarchisée autour des élites de la minorité et des représentants de l'État turc, transmission du statut de notables, cooptation) et le processus externe d'ouverture croissante de la société grecque à la minorité dans le contexte européen. Le contrôle social et familial permet le maintien relatif des frontières du groupe (processus interne), alors que, politiquement, la mobilisation de type communautaire échoue (processus externe). 